\title{
Cintilografia renal com ácido dimercaptossuccínico marcado com tecnécio no diagnóstico da pielonefrite na infância: estudo de 17 casos
}

\author{
Renal scintigraphy using technetium dimercaptosuccinic acid \\ in the diagnosis of pyelonephritis in children: study of 17 cases
}

\section{Eliana B.M. Guidoni' ${ }^{1}$, Marilia M.S. Maroni ${ }^{2}$, Igor M. Mimica ${ }^{3}$, Julio Toporovski ${ }^{4}$}

\section{Resumo}

Objetivo: a infecção do trato urinário é uma das doenças bacterianas mais freqüentes em pediatria. O envolvimento infeccioso renal (pielonefrite) pode evoluir com formação de cicatriz renal irreversível com conseqüente perda funcional e dano renal progressivo. Estudos recentes sugerem que até 50-60\% das cicatrizes renais poderiam ser evitadas ou atenuadas com o diagnóstico precoce da pielonefrite. O objetivo deste estudo é determinar se a presença de alterações na cintilografia renal com DMSA na fase aguda da infecção urinária pode ser indicadora de pielonefrite.

Métodos: empregamos a técnica de washout em 17 meninas portadoras de infecção urinária como padrão-ouro na localização do sítio da infecção. Todas as crianças realizaram cintilografia renal com DMSA na fase aguda da infecção. Os resultados foram analisados pelo teste do qui-quadrado ou de Fisher.

Resultados: a cintilografia renal com DMSA detectou alterações em todos os cinco casos de pielonefrite diagnosticados pelo washout sugerindo envolvimento renal. Observamos também que apenas uma das crianças com cistite (total = 12 casos) apresentava alterações ao DMSA. Portanto, a sensibilidade e especificidade deste estudo com DMSA na fase aguda da infecção foi de $100 \%$ e $92 \%$ respectivamente no diagnóstico da pielonefrite.

Conclusões: a cintilografia renal com DMSA mostrou-se método sensível no diagnóstico da pielonefrite aguda em crianças.

J Pediatr (Rio J) 2001; 77 (2): 119-23: pielonefrite, DMSA, cintilografia, criança.

\section{Introdução}

Definem-se três tipos de ITU de acordo com sua localização e repercussão no trato urinário: bacteriúria assinto-

\footnotetext{
1. Assistente do Serviço de Nefrologia Infantil e Mestre em Pediatria pela Fac. de Ciências Médicas da Santa Casa de São Paulo (FCM-SCSP).

2. Professora Assistente do Departamento de Clínica Médica e Diretora do Serviço de Medicina Nuclear da Santa Casa de São Paulo.

3. Chefe do Serviço de Microbiologia da FCM-SCSP.

4. Chefe do Serviço de Nefrologia Infantil da Santa Casa de São Paulo.
}

\begin{abstract}
Objective: to determine whether the presence of abnormal results in DMSA renal scintigraphy indicates pyelonephritis.

Methods: we performed the washout test in 17 children with urinary tract infection, as a criterion standard, to locate the infection site. All the children underwent DMSA renal scintigraphy in the acute phase of the disease. The results were analyzed by the chisquare test or Fisher test.
\end{abstract}

Results: DMSA renal scintigraphy revealed changes in all five cases of pyelonephritis, suggesting acute kidney involvement. On the other hand, only one child with cystitis (total $=12$ cases) had abnormal results in renal scintigraphy. Sensitivity and specificity were $100 \%$ and $92 \%$, respectively.

Conclusion: DMSA renal scintigraphy is a sensitive method for the diagnosis of pyelonephritis in children.

J Pediatr (Rio J) 2001; 77 (2): 119-23: pyelonephritis, DMSA, scintigraphy, child.

mática, cistite e pielonefrite aguda (PNA). A bacteriúria assintomática e a cistite são infecções benignas, pois não acarretam prejuízo à função renal. A pielonefrite, porém, é particularmente importante, podendo acarretar danos renais progressivos e irreversíveis ${ }^{1-3}$.

Os parâmetros clínicos de localização da ITU (cistite ou pielonefrite) são, em geral, pouco específicos e de difícil 
detecção, principalmente em faixas etárias precoces, em que prevalece o risco de PNA com formação de cicatriz renal.

Métodos diretos e indiretos podem ser utilizados na localização da infecção no trato urinário. Dentre os métodos diretos destacamos a técnica de washout. Esta técnica, descrita pela primeira vez por Fairley et al., em $1967^{3}$, e modificada em $1971^{5}$, consiste em lavagem vesical utilizando-se solução com antibiótico não absorvível para esterilização do trato urinário inferior, seguida de lavagens sucessivas com solução fisiológica para remoção da substância antibacteriana e coleta de amostras de urina para cultura. Após a esterilização vesical transitória, a persistência de uroculturas positivas seria resultado de contaminação de urina proveniente dos ureteres e, portanto, indicativa de PNA. Considerado um método simples, quando aplicado em adultos, apresenta dificuldades técnicas em crianças, relacionadas, principalmente, com a baixa faixa etária e a necessidade de cooperação durante o procedimento. Tem duração longa (cerca de três horas), é trabalhoso e requer adequada retaguarda laboratorial. Está indicado, preferencialmente, apenas para fins de estudo comparativo, uma vez que dispomos atualmente de métodos menos invasivos no diagnóstico da pielonefrite.

Vários métodos indiretos podem ser utilizados na localização do sítio da ITU, tais como diminuição transitória da capacidade de concentração urinária, elevação das enzimas urinárias (desidrogenase lática, beta-glucoronidase, $\mathrm{N}$-acetil-beta-D-glucosaminidase e beta 2-microglobulina), resposta imunológica a infecção (velocidade de hemossedimentação, interleucina 6 e 8, proteína $C$ reativa); pesquisa de coated bacteriano; ultra-sonografia renal (USG) e urografia excretora (UGE). Porém, em geral, apresentam baixa sensibilidade e especificidade no diagnóstico da pielonefrite aguda ${ }^{6-10}$.

O diagnóstico por imagem da PNA vem se aprimorando nas últimas décadas, principalmente através do estudo com radioisótopos. Segundo Smellie ${ }^{11}$ o exame com ácido dimercaptossuccínico marcado com tecnécio (DMSA) apresenta várias vantagens em relação à UGE: utiliza radiofármaco que não apresenta reações alérgicas; não necessita de preparo intestinal prévio; envolve menor taxa de exposição à radiação, preservando as gônadas, e as imagens obtidas são de alta resolução, independentemente da faixa etária do paciente.

O DMSA é fixado nas células tubulares proximais e na parte superior da alça Henle, podendo alcançar estas células diretamente pelo fluxo sangüíneo ou pela reabsorção do filtrado glomerular. A captação do DMSA reflete a integridade funcional do parênquima renal, principalmente no que se refere a córtex.Na PNA, a concentração do DMSA pode estar afetada por alteração no fluxo intra-renal ou na reabsorção tubular proximal ${ }^{12}$. Durante o processo de pielonefrite aguda encontramos hipocaptação do radioisótopo de forma focal ou difusa, uni ou bilateral, que poderá ser acompanhada de aumento do volume renal, como é obser- vado na USG, ou atenuação do contorno renal sem, contudo, apresentar deformidade. Essas alterações secundárias ao processo inflamatório podem persistir de quatro a seis semanas após o tratamento da PNA, sem serem, necessariamente, definitivas. Quando detectadas precocemente pelo exame com DMSA, as alterações encontradas na fase aguda poderão desaparecer em 50 a $60 \%$ dos pacientes adequadamente tratados ${ }^{13-16}$.

$\mathrm{Na}$ última década vários trabalhos têm estudado as alterações encontradas na cintilografia renal com DMSA durante a PNA ${ }^{14,16-22}$. Embora esses trabalhos variem em relação à população estudada, concordam nos seguintes aspectos: o dano ao parênquima renal é detectado pelo exame com DMSA durante a infecção aguda na maioria dos pacientes (50 a 80\%) e o exame com DMSA alterado é raro em crianças com diagnóstico de cistite ${ }^{23,24}$.O objetivo deste estudo é analisar se a presença de alterações na cintilografia renal com DMSA na fase aguda da infecção urinária pode ser indicadora de pielonefrite.

\section{Métodos}

Foram estudadas 17 meninas portadoras de infecção urinária, com faixa etária entre três e 13 anos (média $=5,8$ anos), atendidas na Unidade de Nefrologia do Pronto Socorro Infantil da Santa Casa de São Paulo. Todos os procedimentos foram realizados com o consentimento dos pais ou responsáveis, após esclarecimento dos objetivos deste estudo, e aprovados pela comissão de ética médica. Critérios de exclusão: sexo masculino, pela dificuldade no emprego do washout e baixo grupo etário, devido a não cooperação do paciente durante a realização do washout.

$\mathrm{Na}$ fase aguda da ITU realizamos coleta de urina para confirmação da ITU; emprego da técnica de washout após confirmação da ITU e antes da introdução de antibioticoterapia; cintilografia renal com DMSA durante a primeira semana após o diagnóstico da ITU; coleta de urina para urocultura de controle 48 a 72 horas após o término do tratamento medicamentoso.A coleta de urina foi realizada preferencialmente por jato intermediário após assepsia adequada (água e sabão). A sondagem vesical foi o método de escolha nas meninas portadoras de leucorréia ou quando não havia cooperação na coleta por jato intermediário. Nestes casos, a assepsia foi realizada com clorexidina de acordo com o preconizado pelo Serviço de Infecção Hospitalar da Santa Casa de São Paulo. Tomou-se o cuidado, em ambos os procedimentos, de desprezar-se uma fração inicial de urina, amenizando a contaminação por bactérias provenientes da uretra ${ }^{25}$. Todas as amostras de urina coletadas foram encaminhadas ao Laboratório da Disciplina de Microbiologia e Imunologia do Departamento de Patologia da Faculdade de Ciências Médicas da Santa Casa de São Paulo. As urinas colhidas através de JI foram diluídas em 1/ $1000 \mathrm{e}$, por sondagem vesical, em 1/100 para semeadura em 
placa de agar C.L.E.D. (cistina-lactose-eletrólito deficiente) e incubadas à temperatura de 35 a $37^{\circ} \mathrm{C}$. Para leitura, procedeu-se à contagem de colônias multiplicando-se o valor obtido por 100 ou 1000 de acordo com a diluição utilizada.A interpretação dos resultados foi realizada de acordo com os critérios de $\mathrm{Kass}^{26}$, que considera compatível com ITU um valor maior ou igual a $100.000\left(10^{5}\right)$ unidades formadoras de colônias por $\mathrm{ml}(\mathrm{UFC} / \mathrm{ml})$ para urina colhida através de jato intermediário e maior ou igual a $1000\left(10^{3}\right) \mathrm{UFC} / \mathrm{ml}$ para urina colhida através de sondagem vesical.

Para a realização da técnica de washout procedeu-se o esvaziamento vesical completo através de sondagem de demora, com coleta de urina para cultura (tubo 1); repleção vesical com solução de neomicina de $0,2 \%$ seguida por repouso de 40 minutos; lavagem vesical com soro fisiológico a $0,9 \%$; repleção vesical com soro fisiológico a $0,9 \%$ e repouso por 20 minutos, seguido de esvaziamento da bexiga e coleta de urina para cultura (tubo 2). O quarto procedimento é repetido sucessivamente com coleta de urina para cultura nos tubos $3,4,5$ e 6 , respectivamente. $O$ volume total para repleção vesical é variável e depende da idade e da cooperação do paciente, porém situa-se entre $5 \mathrm{a} 10 \mathrm{ml} /$ $\mathrm{kg}$ de peso corpóreo. O procedimento durou, em média, 3 horas. Os resultados do washout são interpretados como positivos (pielonefrite) se as uroculturas mantêm-se positivas em todos os tubos, negativos (cistite) nos casos em que as uroculturas tornaram-se negativas a partir do terceiro ou quarto tubo de urina e, indeterminado quando as uroculturas mantêm-se positivas, porém não apresentam contagem significativa de colônias.

O estudo cintilográfico com DMSA foi realizado pelo Serviço de Medicina Nuclear do Instituto do Câncer Arnaldo Vieira de Carvalho durante a fase aguda da infecção urinária, entre o segundo e o quinto dia após a confirmação laboratorial. O radiofármaco utilizado foi o ácido dimercaptossuccínico marcado com tecnécio 99 metaestável (IPEN, São Paulo) obtido na forma de pertecnetato de sódio em gerador de molibidênio, na dose de $2 \mathrm{mCi}$ por via endovenosa. As imagens foram adquiridas três a seis horas após a administração do radiofármaco nas incidências posterior e oblíquas posteriores. O laudo consensual foi emitido, após avaliação das imagens obtidas, por dois médicos assistentes do Serviço de Medicina Nuclear. Foram consideradas alterações na captação do exame com DMSA compatíveis com PNA, a hipocaptação focal ou difusa no parênquima renal com aumento do volume renal e/ou uma diferença na quantificação relativa, entre os rins, maior que $10 \% 17,27,28$.

Para análise estatística dos resultados foi utilizado o teste do qui-quadrado ou o de Fisher, o nível de significância foi fixado em 0,05 . Os parâmetros estudados também foram avaliados pelos índices de sensibilidade, especificidade, prevalência real, prevalência estimada, valor preditivo positivo, valor preditivo negativo, classificação correta e incorreta.

\section{Resultados}

De acordo com a técnica de washout, das 17 crianças estudadas, encontramos 5 casos de PNA e 12 casos de cistite. A associação entre PNA e alterações na captação da cintilografia renal com DMSA esteve presente em todos os 5 casos, enquanto apenas uma criança com cistite apresentou alterações no estudo com DMSA. A análise pelo teste de Fisher encontrou significância estatística entre a presença de alterações no exame com DMSA e o diagnóstico de PNA. A sensibilidade desta associação foi de $100 \%$ com $92 \%$ de especificidade. Os demais índices também foram muito satisfatórios (Tabela 1).

Tabela 1 - Análise estatística da associação entre a presença de alteração na cintilografia com DMSA na fase evolutiva da ITU e o diagnóstico de PNA (padrão-ouro = washout)

\begin{tabular}{l|c}
\hline Índice & \% \\
\hline Sensibilidade & 100 \\
Especificidade & 92 \\
Prevalência real & 29 \\
Prevalência estimada & 35 \\
Valor preditivo negativo & 100 \\
Valor preditivo positivo & 83 \\
Classificação correta & 94 \\
Classificação incorreta & 6 \\
\hline
\end{tabular}

\section{Discussão}

A técnica de washout foi realizada satisfatoriamente em todos os casos de ITU analisados neste estudo. Durante a realização deste procedimento, verificamos algumas dificuldades e sugerimos algumas recomendações: na repleção vesical a sonda vesical utilizada deve ser adequada à idade da criança, não permitindo escape da solução; a criança deve permanecer tranqüila durante todo o período de enchimento vesical para que o procedimento seja realizado satisfatoriamente, e o enchimento vesical seja lento, para não despertar precocemente o reflexo de micção; no repouso devemos observar que a criança permaneça deitada e tranqüila durante todo o período de repouso de cada solução na bexiga, para que se obtenha o efeito desejado e, durante o esvaziamento vesical, a mudança de decúbito ou a colocação da criança em posição supina pode facilitar a drenagem vesical completa. É aconselhável, e julgamos de fundamental importância, que a mãe ou responsável permaneça com a criança até o término do exame. As coletas de 
urina para cultura devem ser realizadas preferencialmente no final de cada drenagem vesical. Salienta-se que, em nosso estudo, todos os resultados obtidos com o emprego desta técnica puderam ser classificados em washout positivo (PNA) ou negativo (cistite) não tendo sido observados casos indeterminados.

No presente estudo, das 17 meninas submetidas à técnica de washout, encontramos cinco casos de PNA e 12 casos de cistite. Observamos que, na fase aguda da infecção, todas as cinco crianças portadoras de PNA apresentaram alterações na cintilografia sugestivas de infecção renal aguda, com sensibilidade de $100 \%$ e especificidade de $92 \%$. A hipocaptação focal com preservação do contorno renal associado à queda na captação relativa do radiofármaco pelo rim acometido foi a alteração mais frequiente no exame com DMSA em crianças portadoras de PNA. Estes resultados estão de acordo com os descritos por Rushton ${ }^{12}$.

Nos 11 casos de cistite, verificamos captação normal do DMSA na cintilografia inicial, afastando-se, desta maneira, a hipótese de envolvimento infeccioso do parênquima renal. Entretanto, um caso de cistite apresentou hipocaptação focal do radioisótopo associada a deformidade no contorno renal durante a fase aguda da infecção. A presença de hipocaptação renal do DMSA acompanhada de deformidade é sugestiva de cicatriz pielonefrítica pregressa. Não pudemos afastar, contudo, a possibilidade de novo foco de PNA próximo à área cicatricial, pois, em rins previamente lesados, a área circunvizinha à fibrose torna-se mais suscetível ao refluxo intra-renal, aumentando a extensão da lesão ${ }^{12,29}$. Desse modo, mesmo que, em pequeno número de casos, ocorra resultado falso positivo na cintilografia renal com DMSA, na dúvida em se tratar de processo puramente cicatricial pregresso ou novo foco infeccioso adjacente à cicatriz pré-estabelecida, a terapêutica para PNA está justificada.

A precocidade na visualização, pelo estudo com DMSA, de sinais indicativos de formação da cicatriz renal, particularmente em lactentes e crianças menores de cinco anos de idade, é descrita por diversos autores ${ }^{20-22}$. Rushton \& Majd $^{13}$, acompanhando 33 crianças com PNA por quatro a 42 meses (média $=10,7$ meses), observaram que $42 \%$ dos rins formaram cicatriz renal ou progressão da cicatriz pregressa, enquanto $58 \%$ evoluíram com restituição completa do parênquima renal.

O diagnóstico da PNA continua a representar desafio em crianças portadoras de ITU. As cicatrizes renais advindas do tratamento tardio ou inadequado influem substancialmente no prognóstico da doença renal a longo prazo. Acreditamos que o diagnóstico correto da ITU, a valorização da presença de fatores predisponentes à PNA com eventual formação de cicatrizes e a avaliação renal através do estudo com DMSA poderiam favorecer o tratamento precoce da pielonefrite e seu acompanhamento evolutivo, diminuindo assim, o risco de deterioração da função renal.

\section{Referências bibliográficas}

1. Toporovski J, Mello VR. Infecção do trato urinário na infância (ITU). Rev Paul Pediatr 1984; 2:7-17.

2. Winberg J, Anderson HJ, Bergstrom T, Jacobsson B, Larson H, Lincoln K . Epidemiology of syntomatic urinary tract infection in childhood. Acta Paediatr Scand Suppl 1974; 252:1-20.

3. Winberg J. Urinary tract infections in children. J Infect Dis 1990; 3:55-61.

4. Fairley KF, Bond AG, Brown RB, Habersberger P. Simple test to determine the site of urinary-tract infection. Lancet 1967; 2:427-8.

5. Fairley KF, Carson NE, Gutch RC, Leighton P, Grounds AD, Laird EC, et al. Site of infection in acute tract infection in general pratice. Lancet 1971;2:615-8.

6. Marild S, Wettergren B, Helltrom M, Jodal U, Lincoln K, Orskov $\mathrm{J}$, et al. Bacterial virulence and inflamatory response in infants with febrile urinary tract infection or screening bacteriúria. J Pediatr 1988; 112:348-54.

7. Winberg J, Bollgren J, Kallenius G, Mollby R, Svenson SB. Clinical pyelonephritis and focal renal scarring. A selected review of pathogenesis prevention and prognosis. Pediatr Clin North Am 1982; 29:810-4.

8. Winberg JP. Fimbriae, bacterial adhesion and pielonephritis. Arch Dis Child 1992; 59:180-4.

9. De Man P, Jodal U, Svanborg-Éden C. Dependence among host response parameters used to diagnose urinary tract infection. $\mathrm{J}$ Infect Dis 1991; 163:331-5.

10. Johnson CE, De Baz BP, Shurin PA, De Bartolomeo R. Renal ultrasound evaluation of urinary tract infections in children. Pediatrics 1986; 78:871-8.

11. Smellie JM. The intravenous urogram in the detection and evaluation of renal damage following urinary tract infection. Pediatr. Nephrol 1995; 9:213-9.

12. Rushton HG. The evaluation of acute pyelonephritis and renal scarring with technetium 99M-dimercaptosuccinic acid renal scintigraphy: evolving concepts and future directions. Pediatr. Nephrol 1997; 11:108-20.

13. Rushton HG, Majd M. Dimercaptosuccinic acid renal scintigraphy for the evaluation of pyelonephritis and scarring: a review of experimental and clinical studies. J Urol 1992; 148:1726-32.

14. Bernador D, Bernador N, Slosman DO, Nussle D, Mermillod B, Girardin E. Cortical scintigraphy in the evaluation of renal parenchymal changer in children with pyelonephritis. J Pediatr 1994;124:17-20.

15. Bernador D, Bernador N, Slosman D, Mermillod B, Girardin E. Are younger children at highest risk of renal sequelae after pyelonephritis? Lancet 1997; 349:17-9.

16. Jakobsson B, Nolstedt L, Svensson L, Soberlundh S, Berg U. $99 \mathrm{M}$ technetium-dimercaptosuccinic acid scan in the diagnosis of acute pyelonephritis in children: relation to clinical and radiological findings. Pediatr Nephrol 1992; 6:328-334.

17. Majd M, Rushton HG, Jantaush B, Wiedermann BL. Relationship among vesicoureteral reflux, P-fimbriated Escherichia coli and pyelonephritis in children with febrile urinary tract infection. J Pediatr 1991; 119:578-85.

18. Ditchfield MR, De Campo JF, Cook DJ, Nolan TM, Powell HR, Sloane R, et al. Vericoureteral reflux: an accurate predictor of acute pyielonephritis in childhood urinary tract infection? Radiology 1994 a; 190:413-5.

19. Ditchfield MR, De Campo JF, Nolan TM, Cook DJ, Grimwood $\mathrm{K}$, Powell HR, et al. Risk factors in the development of early renal cortical defects in children with urinary tract infection. ARJ Am J Roentgenol 1994b; 162:1393-7. 
20. Goldraich NP, Ramos OL, Goldraich JH. Urography versus DMSA scan in children with vesicoureteric reflux. Pediatr Nephrol 1995; 9:221-6.

21. Goldraich NP, Goldraich JH. Update on dimercaptosuccinic acid renal scanning in children with urinary tract infection. Pediatr Nephrol 1995; 9:221-6.

22. Pahl MMC. Infecção urinária na infância: alterações renais na fase aguda e na evolução, detectadas pela cintilografia com ácido dimercaptossuccínico marcado com tecnécio-99M (99mTc DMSA) [tese]. São Paulo: Escola Paulista de Medicina; 1998.

23. Tappin DM, Murphy AV, Mocan H, Shaw R, Beeattie TJ, Mc Allister TA, Mac Kenzi JR. A prospective study of children with fisrt acute symptomatic E. coli urinary tract infection. Early 99m-technetium dimercaptosuccinic acid scan appearances. Acta Paediatr Scand 1989; 78:923-9.

24. Verboven M, Ingels M, Delree M, Piepz A. 99mTc-DMSA scintilography in acute urinary tract infection in children. Ped Rad 1990; 20:540-2.

25. Marino MDV. Avaliação de métodos laboratoriais no diagnóstico das infecções urinárias [tese]. São Paulo: Faculdade de Ciências Médicas da Santa Casa de São Paulo, 1997.

26. Kass EB. Bacteriuria and the diagnosis of infections of the urinary tract. Arch Intern Med 1957; 100:709-14.
27. Daly MJ, Jonas WA, Rudd TG, Tremann JA - Differential TC99 dimercaptosuccinic acid (DMSA) renal localization: correlation with renal function. J Nucl Med 1977; 18:594-5.

28. Daly MJ, Jones W, Rudd TG, Tremann J. Differential renal function using technetium-99m dimercaptosuccinic acid (DMSA): in vitro correlation. J Nucl Med 1979; 20:63-6.

29. Ransley PG, Risdon RA. Renal papillary morphology and intrarenal reflux in young pig. Urol Res 1975; 3:105-9.

Endereço para correspondência:

Dra. Eliana B.M. Guidoni

Rua Loefgreen, 1654/53

São Paulo - SP - CEP 04040-002

Fone: (11) 5571.9071 